\title{
Multiple imputation of multiple multi-item scales when a full imputation model is infeasible
}

\author{
Catrin O. Plumpton ${ }^{1 *}$, Tim Morris ${ }^{2,3}$, Dyfrig A. Hughes ${ }^{1}$ and lan R. White ${ }^{4}$
}

\begin{abstract}
Background: Missing data in a large scale survey presents major challenges. We focus on performing multiple imputation by chained equations when data contain multiple incomplete multi-item scales. Recent authors have proposed imputing such data at the level of the individual item, but this can lead to infeasibly large imputation models.

Methods: We use data gathered from a large multinational survey, where analysis uses separate logistic regression models in each of nine country-specific data sets. In these data, applying multiple imputation by chained equations to the individual scale items is computationally infeasible. We propose an adaptation of multiple imputation by chained equations which imputes the individual scale items but reduces the number of variables in the imputation models by replacing most scale items with scale summary scores. We evaluate the feasibility of the proposed approach and compare it with a complete case analysis. We perform a simulation study to compare the proposed method with alternative approaches: we do this in a simplified setting to allow comparison with the full imputation model.
\end{abstract}

Results: For the case study, the proposed approach reduces the size of the prediction models from 134 predictors to a maximum of 72 and makes multiple imputation by chained equations computationally feasible. Distributions of imputed data are seen to be consistent with observed data. Results from the regression analysis with multiple imputation are similar to, but more precise than, results for complete case analysis; for the same regression models a $39 \%$ reduction in the standard error is observed. The simulation shows that our proposed method can perform comparably against the alternatives.

Conclusions: By substantially reducing imputation model sizes, our adaptation makes multiple imputation feasible for large scale survey data with multiple multi-item scales. For the data considered, analysis of the multiply imputed data shows greater power and efficiency than complete case analysis. The adaptation of multiple imputation makes better use of available data and can yield substantively different results from simpler techniques.

Keywords: Missing data, Multiple imputation, Multi-item scale, Survey data

\section{Background}

Missing data is ubiquitous in research, and survey data is particularly prone to incomplete responses. One problem arising from missing data is a loss of precision and

\footnotetext{
*Correspondence: c.o.plumpton@bangor.ac.uk

${ }^{1}$ Centre for Health Economics and Medicines Evaluation, Bangor University, Ardudwy, Normal Site, Holyhead Road, Bangor, Gwynedd LL57 2PZ, UK

Full list of author information is available at the end of the article
}

statistical power. However, poor handling of the missing data during analysis can lead to biased results.

When handling missing data, assumptions must be made about the mechanism of missingness; no analysis with missing data is free of such assumptions. Data may be missing completely at random (MCAR), where the probability of missing data is not dependent on either the observed or unobserved data. When data is missing at random (MAR), the probability of the data being missing does not depend upon the unobserved data, but 
missingness may be related to the observed data. Alternatively, data may be missing not at random (MNAR), whereby missingness is dependent upon the values of the unobserved data, conditional on the observed data [1-3].

Roth, in 1994, stated that despite its importance, conspicuously little research on missing data analysis appeared within the social sciences literature [4]. It is acknowledged that a gap still exists between techniques recommended by methodological literature and those employed in practice; traditional ad-hoc techniques such as deletion and single imputation techniques are still applied routinely $[3,5,6]$.

Complete case (CC) analysis is commonly used, and is efficient and valid under MAR, provided missing data occurs only in the outcome. Once missing data occurs in covariates, or in parts of a composite outcome, complete case analyses are inefficient. Also, when the MCAR assumption does not hold, the data is no longer representative of the target population, compromising external generalisability [7].

Modern missing data methodologies include maximum-likelihood estimation (MLE) methods such as expectation-maximisation (EM) and multiple imputation (MI), both recommended for data which is MAR [3]. MI has been shown to be robust under departures from normality, in cases of low sample size, and when the proportion of missing data is high [2]. With complete outcome variables, $\mathrm{MI}$ is typically less computationally expensive than MLE, and MLE tends to be problem-specific with a different model being required for each analysis [8].

Whilst many theoretical works suggest MI to be an appropriate method, it has only recently been widely applied in practice [9]. Reviews on handling missing data across different fields indicate that it is relatively rare that missing data, and how it is handled, are reported explicitly: in cost-effectiveness analysis $22 \%$ of studies did not explicitly report missing data [10]; in education the corresponding figure is $31 \%$ [11]; in cohort studies $16 \%$ of studies did not report how much data was missing whilst $14 \%$ of studies did not report how missingness was handled [12]; in epidemiology $46 \%$ of studies were unclear about the type of missing data [13]; and in applied education and psychology $66 \%$ of studies where the presence of missing data could be inferred did not mention missing data explicitly [6]. A review of randomised controlled trials identified 77 articles from the latter half of 2013, of which 73 reported missing data. Of these articles, $45 \%$ performed complete case analysis, $27 \%$ performed simple imputation (linear interpolation, worst case imputation or last observation carried forward) and only $8 \%$ used multiple imputation [14]. Whilst MI and MLE are gaining popularity, ad-hoc techniques still appear in the applied literature, with complete case analysis remaining as the most popular approach.

Large scale survey data presents a number of challenges to imputation: a high number of variables; complexity of the data set; categorical (non-Normal) variables; categories with low observed frequency (sparsity in responses); questions which are conditional upon previous responses; and multiple multi-item scales, which are summed (either directly or weighted) during analysis. Such challenges reduce the use of sophisticated imputation techniques. As missing data in a single item of a multi-item scale leads to a missing total, the rate of missing data in scale totals can be very high. Imputing at the level of scale total whilst ignoring individual items may therefore introduce unnecessary bias. The widelyused EQ 5D-3L is one such scale, consisting of 5 items. A recent study considered imputing at item level rather than imputing scale totals [15]. When the pattern of missingness tended towards all items being missing for a respondent, little difference was seen between methods. When the pattern of missingness tended towards individual items being missing, for sample sizes of $n>100$, imputing at item level was shown to be more accurate.

Another study proposed methods for handling multiitem scales at the item score level [16], and further emphasised how mean imputation or single imputation leads to bias and underestimation of standard errors. The study concludes that missing data should be handled by applying multiple imputation to the individual items. However, the size and complexity of large survey data can cause complete MI prediction models to fail to converge when the model is specified at item level, rendering the ideal method computationally infeasible.

The present study aims to develop an imputation method which addresses the challenges presented by large scale survey data, reducing the size of the prediction model whilst allowing for item level imputation. A simulation study presents a comparison of our proposed method with alternative imputation approaches, and the proposed method is illustrated further using data from a large multinational survey as a case study.

\section{Methods}

\section{Multiple imputation by chained equations}

Multiple imputation for a single incomplete variable works by constructing an imputation model relating the incomplete variable to other variables and drawing from the posterior predictive distribution of the missing data conditional on the observed data [1]. The approach allows for uncertainty in the missing data values by introducing variability in the imputed items.

To handle multiple incomplete variables we use multiple imputation by chained equations (MICE) which allows 
different variable types (continuous, nominal, ordered categorical) to be handled within the same data set [1].

In MICE, variables are initially ordered by level of missingness. Missing values are initially replaced for each variable, for example by drawing at random from the observed values of that variable. Imputation is then conducted on each variable sequentially using the observed and currently imputed values of all other variables in the imputation model. In order to stabilise, this imputation step (known as a cycle) is repeated (typically 10 times) to produce one imputed data set. The process is repeated until the desired number of imputed data sets is reached $[1,17]$.

\section{Imputation using subscale totals}

Often, survey data contains responses to multiple multiitem scales. Imputing every item individually may lead to an unwieldy imputation model, which in extreme cases may fail to converge. In order to reduce the size of the imputation models yet retain item level imputation (and not discard data), we propose to impute responses to individual scale items, using the scale totals within prediction equations. In addition, when imputing responses to an item which forms part of a multiple multi-item scale, responses to other items from the scale should also be included.

As a simple example, suppose we have primary outcome measure $p, \mathrm{n}$ demographic variables $\left(d_{1} \ldots d_{n}\right)$, a multi-item scale $\mathrm{S}$ made up of 7 items $\left(s_{1} \ldots s_{7}\right)$, and a multi-item scale $\mathrm{T}$ made up of 17 items $\left(t_{1} \ldots t_{17}\right)$.

The forms of the imputation models are:

- $d_{1}$ is imputed using the observed and current imputed values of $p, d_{2} \ldots d_{n}$, $s$ and $t$, where $s$ and $t$ are the summed scale scores of $S$ and $T$.

- $s_{1}$ is imputed using the observed and current imputed values of $p, d_{1} \ldots d_{n}, s_{2} \ldots s_{7}$ and $t$.

- $s_{2}$ is imputed using the observed and current imputed values of $p, d_{1} \ldots d_{n}, \mathrm{~s}_{1}, s_{3} \ldots s_{7}$, and $t$.

- $t_{1}$ is imputed using the observed and current imputed values of $p, d_{1} \ldots d_{n}, s$ and $t_{2} \ldots t_{17}$.

- $t_{2}$ is imputed using the observed and current imputed values of $p, d_{1} \ldots d_{n}, s, t_{1}$ and $t_{3} \ldots t_{17}$.

with similar imputation models for $d_{2} \ldots d_{n}, s_{2} \ldots s_{7}$ and $t_{3} \ldots t_{17}$. The proposed approach condenses information from other scales to reduce the number of predictors in each equation. Subscale totals are recalculated after each cycle of imputation.

\section{Categorical variables}

Survey data typically contains categorical variables, which may be either nominal or ordered. Ordered categorical variables, often in the form of Likert scales, can be imputed using ordinal logistic regression, whilst nominal categorical variables may be imputed using multinomial logistic regression. Sparsity may cause nonconvergence errors during multinomial logistic regression, a recognised problem $[1,18]$. On occasion, this may require response categories to be collapsed prior to imputation.

\section{Conditional imputation}

Survey design may contain some conditional questions. For example a question on experience of a specific drug will only be relevant to someone who has taken it. Within the statistical package, Stata, multiple imputation has options for conditional imputation within the -ice- routine [19]. Responses to the second part of the question are only imputed, given a certain answer to the first part of the question.

\section{Analysing multiply imputed data}

During analysis, each of the $M$ imputed data sets are analysed individually. Imputation-specific coefficients are then pooled using Rubin's rules, to produce a single result [20]. Rubin's rules allow the incorporation of both within imputation variance (accounting for uncertainty if the data were complete), and between imputation variance (accounting for uncertainty about the missing data) [1].

\section{Case study}

Our data comes from an online survey, designed to investigate associations between putative predictors of adherence to antihypertensive medication, and patients' self-reported adherence. Detailed methods of the survey and the main findings are published elsewhere [21]. Briefly, cross-sectional survey data from 2595 respondents from nine European countries (Poland, Wales, England, Hungary, Austria, Germany, Greece, the Netherlands and Belgium) was collected using the online tool SurveyMonkey ${ }^{\circledR}$. The target population was adult hypertensive patients who have been prescribed antihypertensive medication.

The survey comprised 13 validated measures from health psychology and behavioural economics, alongside demographic questions, resulting in a total of 135 questions. Within the health psychology sections, responses to several questions were summed to form subscale totals, as per validated approaches to analysing these measures. There are a total of 14 such subscales within the survey. Due to the length of the survey, a level of missing data was to be expected, with respondents dropping out part way through or skipping one or more question. We ensured no missingness in the primary outcome measure, the Morisky measure of adherence [22], by 
enabling 'forced answer' settings within SurveyMonkey. Figure 1 presents the percentage of complete responses by question, and in the order the questions were asked. A dip is seen at the open ended time preference measure, which may be perceived as cognitively challenging [23]. The sensitivity of information requested on income explains the final dip in the plot. Missing data was assumed to be MAR. We consider the impact of possible departures from MAR in the discussion.

We chose to impute each country-specific data set separately, as associations between variables were expected to differ between countries.

A complete MI prediction equation results in 134 predictors for each incomplete variable. Some categorical variables had categories with low observed frequency which presented additional challenges. These were handled by collapsing response categories. Taking education as an example, in Greece, $52.3 \%$ received primary education as a highest educational attainment, $29.0 \%$ secondary education and $18.7 \%$ higher education. For England the corresponding figures were $0.3,33.7$ and $65.3 \%$ respectively. We collapsed the lower two categories, conducting the final analysis on 'up to secondary education' and 'higher education'. Collapsing of categories was applied to all data sets, and was maintained for analysis.
Within the income section of the survey, questions had an 'opt out' response if respondents were unwilling to provide the information. Additional file 1: Table S1 summarises these responses across the nine countries. Questions in this section took an ordered categorical format, which we were keen to preserve (rather than impute as nominal variables). This was achieved by generating two separate variables for each income item. An initial binary variable reflected whether the respondent was willing to provide a response. An ordinal variable then reflected the response, conditional on the respondent being willing to provide the information.

The number of imputations, $M$, was chosen based upon analysis of the Polish data set, which was received 3 months prior to data from other countries. For this data set $26 \%$ of response values were missing, so the number of imputations was set at $M=25$, closely reflecting the suggestion of one imputation per percent missing data [1]. For subsequent country-level data sets the amount of missing data was in fact lower, 5-22 \%, but $M=25$ was maintained for consistency.

Initially a full imputation model was attempted for this data, but failed to converge for all imputations. Applying our proposed approach, model size depended on the number of items within each subscale (range, 56-72).

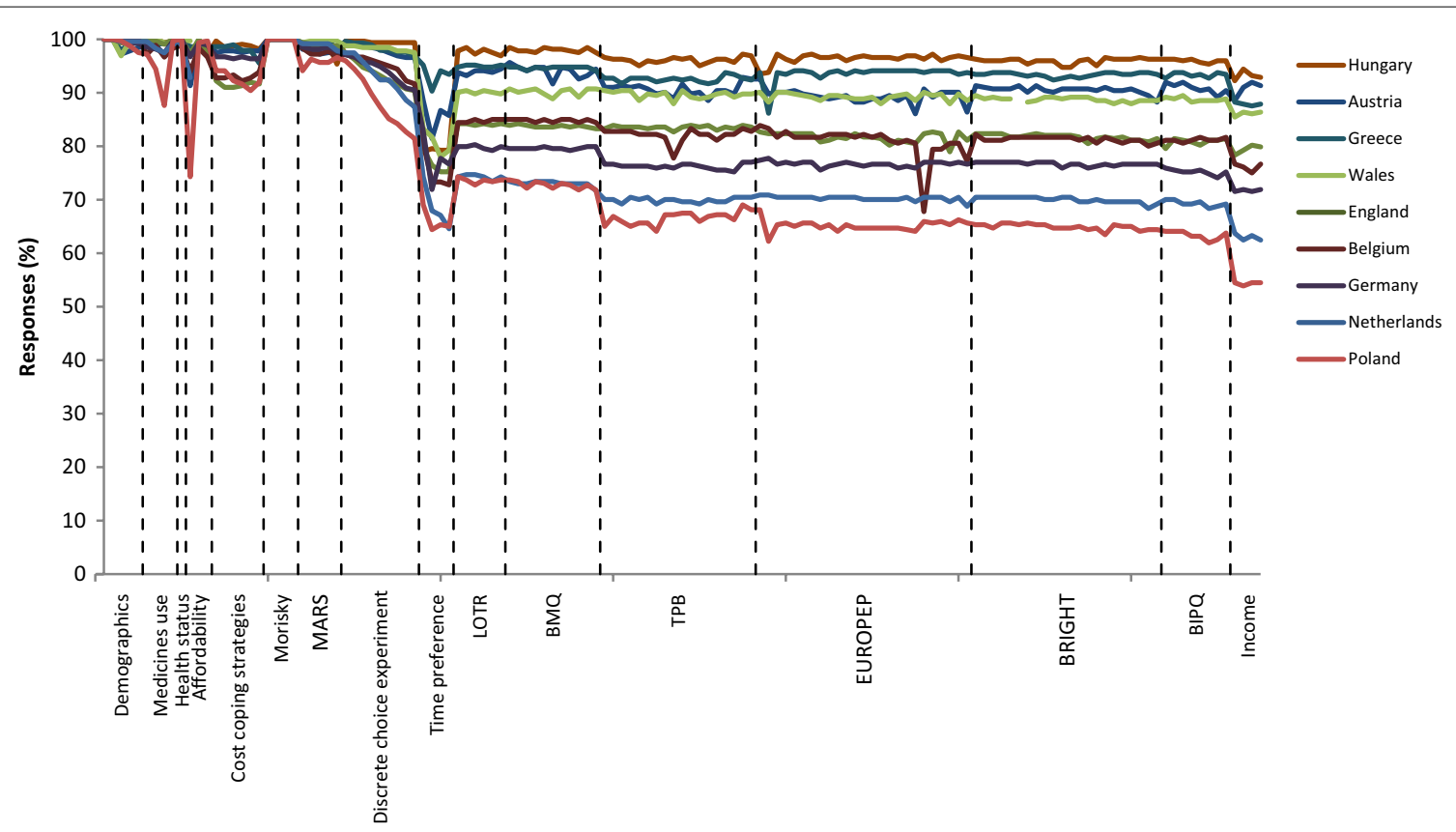

Fig. 1 Responses rate by question and country. Questions not-applicable due differences in healthcare systems appear as breaks in the plot. MARS Medication Adherence Rating Scale, LOTR Life Orientation Test Revised, BMQ Beliefs about Medicines Questionnaire, TPB Theory of Planned Behaviour, EUROPEP European Task Force on Patient Evaluations of General Practice, BRIGHT Building Research Initiative Group IIIness Management and Adherence in Transplantation, BIPQ Brief Illness Perception Questionnaire Reprinted from Value in Health, 18(2), Morrison VL, Holmes EAF, Parveen S, Plumpton CO, Clyne W, De Geest S, Dobbels F, Vrijens B, Kardas P, Hughes DA, Predictors of Self-Reported Adherence to Antihypertensive Medicines: A Multinational, Cross-Sectional Survey, 206-216, Copyright (2015), with permission from Elsevier [21] 
Each prediction equation included the demographic variables and the primary outcome measure. MI was conducted using the -ice- routine in Stata 10 [19, 24, 25]. Categorical variables were handled using -mlogit- and -ologit- [25]. Subscale totals were calculated following each cycle of the imputation using the passive option of the -ice- routine. The final imputation methods and models used to impute different parts of the survey are summarised in Table 1, with an extract of Stata code provided in Additional file 2: Appendix S1.

\section{Data analysis}

Primary analysis was to be conducted by country, and the survey was powered as such. The primary analysis was a logistic regression with Morisky score as outcome, aiming to identify predictors of non-adherence to medication. There were deemed to be too many predictors to enter into the model, $\mathrm{N}=42$ (Table 1), therefore an initial variable selection step was employed. For the regression results presented here, we have used the same pragmatic variable selection as in the main analysis [21]: continuous variables were selected using univariate tests, pooled using Rubin's rules; categorical variables were selected using Chi squared tests and ANOVA on complete case data; and variables relating to numbers of medicines were selected using t-tests controlling for age on complete case data. Variables showing univariate significance with the outcome measure were entered into the regression model.

We also compared variable selection using complete case data with variable selection procedure using Rubin's rules in the pooled MI data, using unadjusted or ageadjusted analyses as described above [20,26].

\section{Simulation}

We devised a simulation study to impartially assess the performance of the new method against some alternatives in a realistic setting-based on the case study.

We invoked a simpler set up than the case study, to allow comparison of the proposed strategy with a full imputation model, which is not possible on the full data set. The variables included were Morisky score (fully observed), age in years (fully observed), attitude (partially observed, the sum of seven items, scored as integers from 1 to 5) and practitioner satisfaction (partially observed, the sum of 17 items, also integers from 1 to 5). We estimated four quantities: the means of attitude and practitioner satisfaction, and their coefficients in a logistic regression of Morisky score on age, attitude and practitioner satisfaction.

\section{Simulation procedure}

The simulation procedure was as follows for 1000 replications:
- 323 observations of the 26 variables (Morisky score, age, 7 items of attitude, 17 items of practitioner satisfaction) were simulated from a multivariate normal distribution based on the observed vectors of means and standard deviations and the observed correlation matrix.

- Morisky score was rounded to the nearest of 0 or 1 . Items making up attitude and practitioner satisfaction were rounded to take values of 1-5.

- Missing values were introduced for items of the attitude and practitioner satisfaction scales. The probability of missing data depended on Morisky score and age, based on the real dataset (MAR). Each observation was assigned to one of three categories: all items observed, some items observed, or no items observed. Three scenarios are simulated:

- Base case: $35 \%$ had all items missing for a scale; $8 \%$ had one or two items missing.

- More incomplete observations with partial data: $18 \%$ had all items missing for a scale; $25 \%$ had one or two items missing.

- Fewer observations with complete data: $55 \%$ had all items missing for a scale; $15 \%$ had just one or two missing.

For each simulated dataset six methods were considered for dealing with the missing data, presented in Table 2. Ten imputations were used for all MI-based approaches.

\section{Outcomes}

For each parameter of interest we summarise percent bias (compared to analysis of complete data), coverage, and efficiency (through the empirical standard error, expressed by comparison to method 1) over the 1000 replications for that scenario. Estimates are accompanied by Monte-Carlo $95 \%$ confidence intervals.

\section{Results \\ Case study}

To compare the performance and fit of the MI models, we plot complete case data versus imputed data, overall and by imputation. Figures 2 and 3 illustrate such comparisons for the individual item and scale total which displayed the highest proportion of missing data. These are one of the time preference variables (36\% missing data), and the support scale (70\% of scale totals missing, $43 \%$ individual items missing), both from the Polish data set. On inspection, in both cases, the imputed data is similar but not identical to the complete case data.

For those variables which were entered into the regression model in five or more countries, the regression 


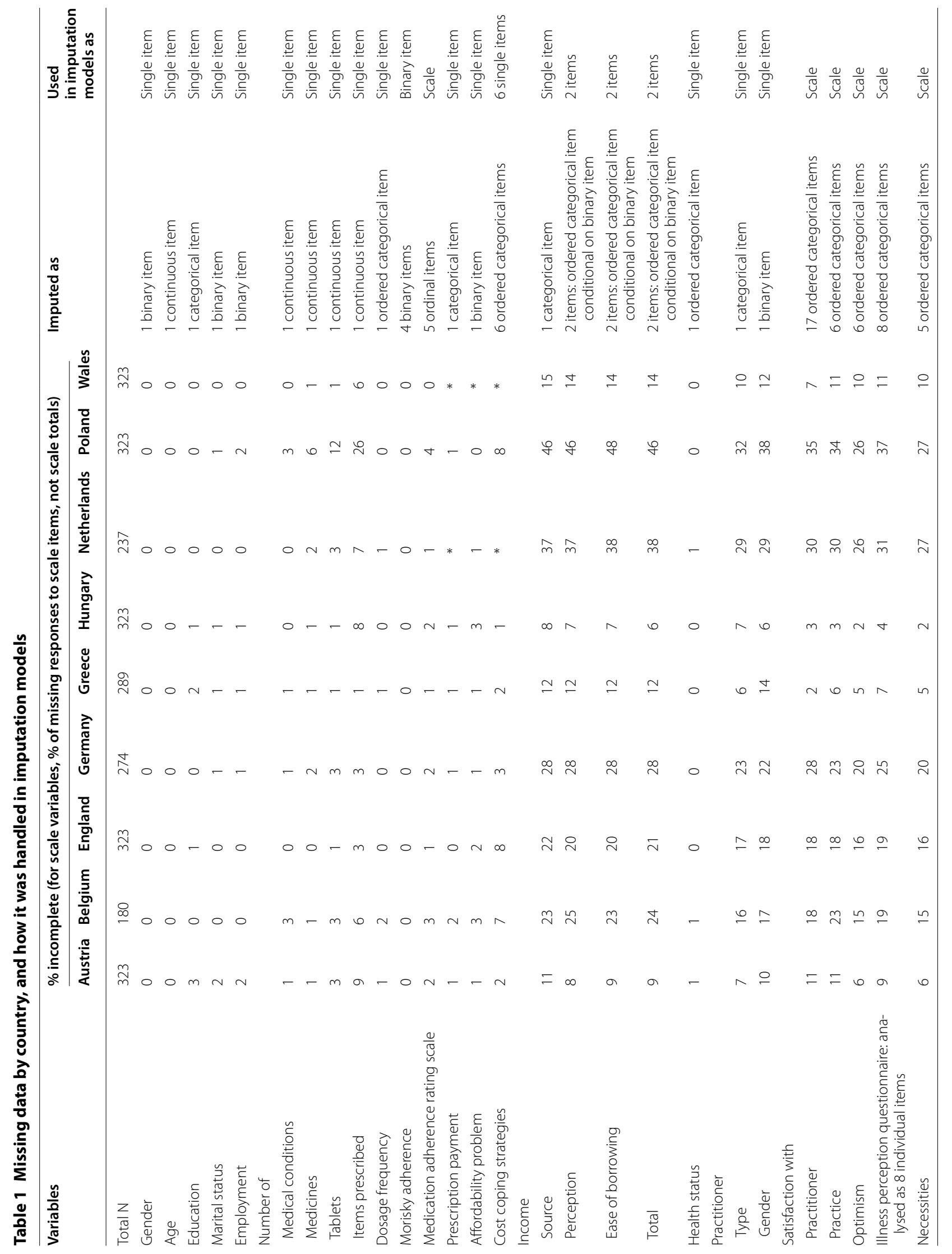




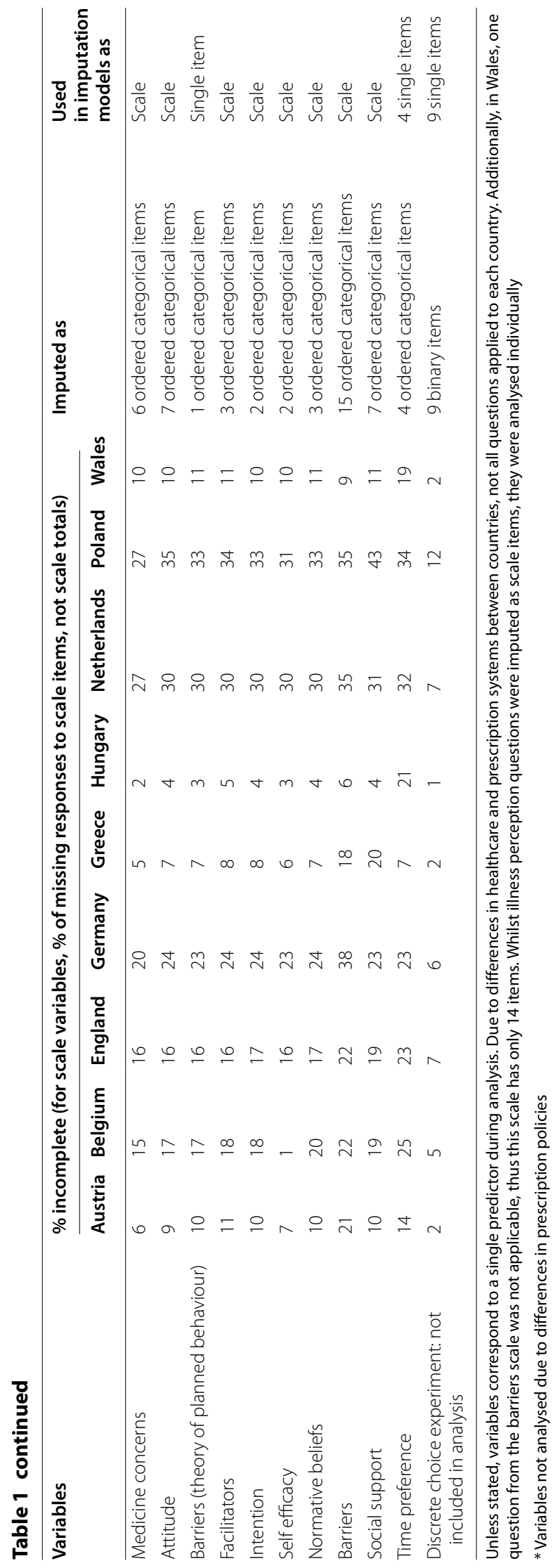




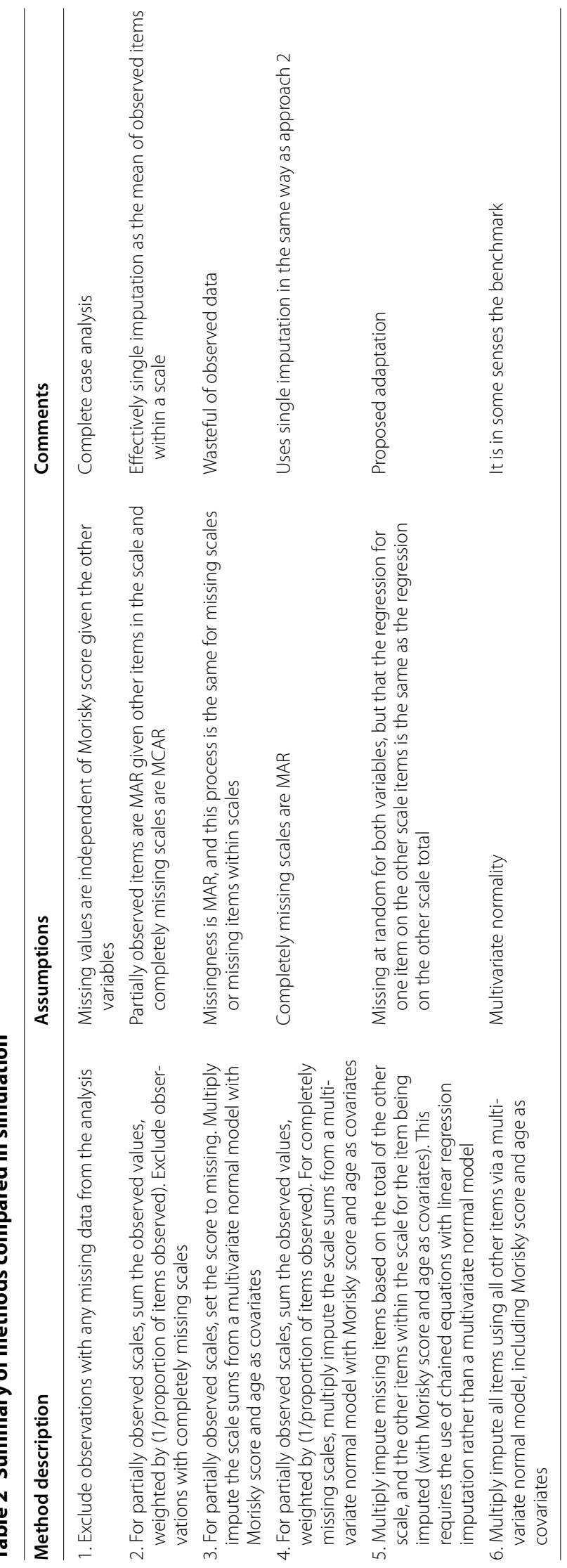




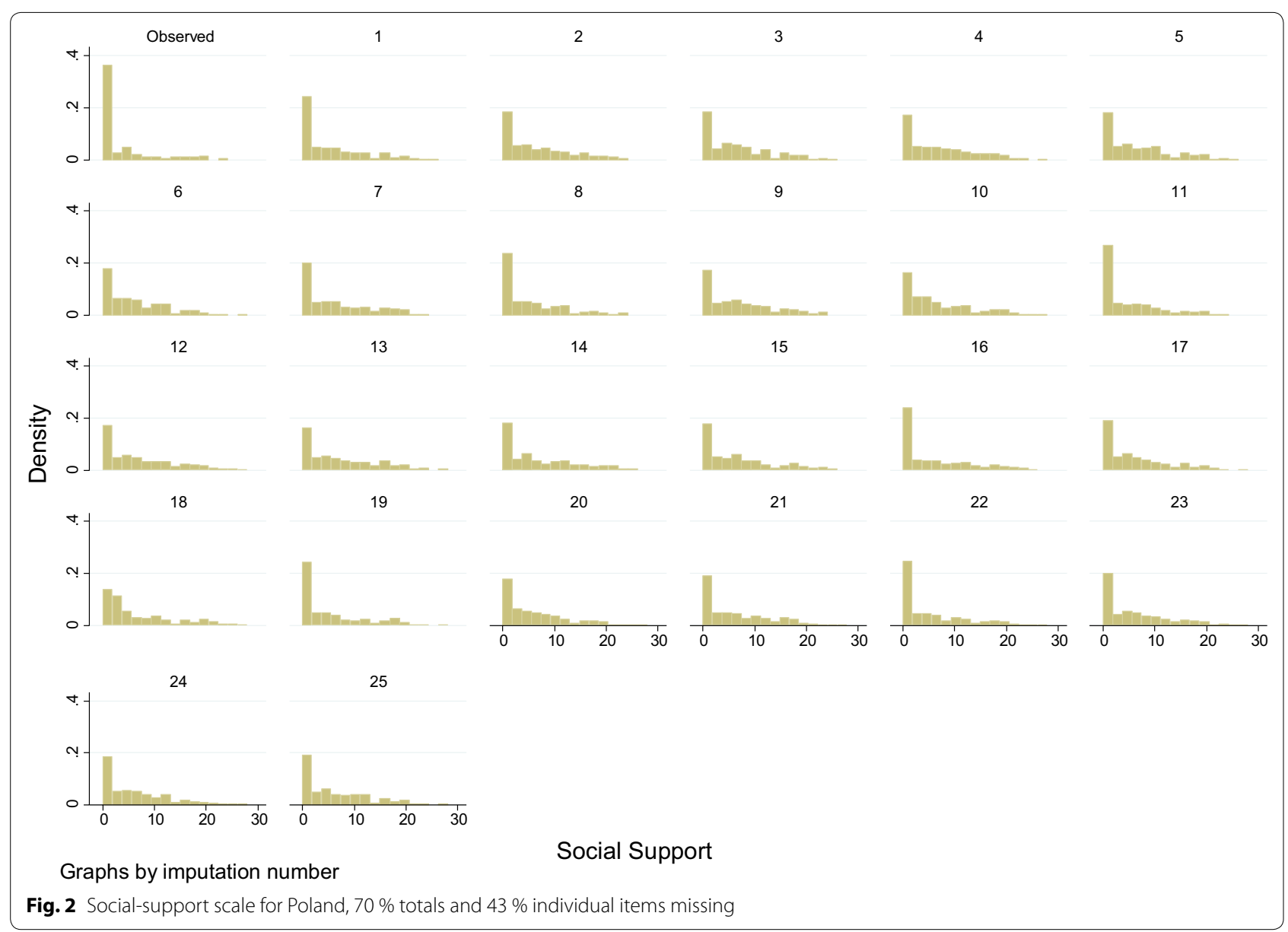

results are illustrated as odds ratios with $95 \%$ confidence intervals in Fig. 4. Differences in the significance of results are observed between data analysed using MI and CC for age, barriers and personal control in Austria, barriers and self-efficacy in England, barriers and employment in Poland and age in Wales. The majority of differences (except barriers in Austria and Poland) are attributable to narrower confidence intervals in the MI analysis, thus illustrating the higher power and efficiency which the MI approach offers. Whilst differences in the standard errors alter the significance of the results, there are no substantial differences in the point estimates of the $\beta$-coefficients.

Table 3 presents the proportional reduction in standard error for MI compared to CC analysis, summarised for all variables entered in the country level regression analyses. From the table, it can be seen that on average, standard error is reduced by $39 \%$ when an MI approach is adopted over CC analysis. Standard errors are smaller for MI than CC analysis for all variables other than illness coherence in Belgium (where there was no change in standard error between MI and CC). To ensure that reduction in standard error was not biased by variable selection method, reduction was also compared for variables selected using CC and MI approaches. For variables selected using the $\mathrm{CC}$ method, mean standard error reduction was $45 \%$ (95\% CI: 12, $78 \%$; range $15-99.9 \%$ ). For variables selected using the MI method, mean standard error reduction was $34 \%$ (95\% CI: 11, 58 \%; range $2-57 \%)$.

For the univariate variable selection, disparity between which variables were selected using either the MI or CC approach is summarised in Table 4. Chi squared tests indicate that the disparity was significant, $\left(X^{2}=250\right.$, $\mathrm{p}<0.001$ ), with agreement (sum of the main diagonal) achieved for only $92.5 \%$ of variables. Lower agreement is observed in the variables with more missing data: at $<20 \%$ missing data agreement was $94 \%$, compared to $88 \%$ when missing data was $>20 \%$.

\section{Simulation}

Figures 5, 6 and 7 show the results of the simulation for the three scenarios. In the base case Fig. 5, significant downward bias is seen for the mean of practitioner 


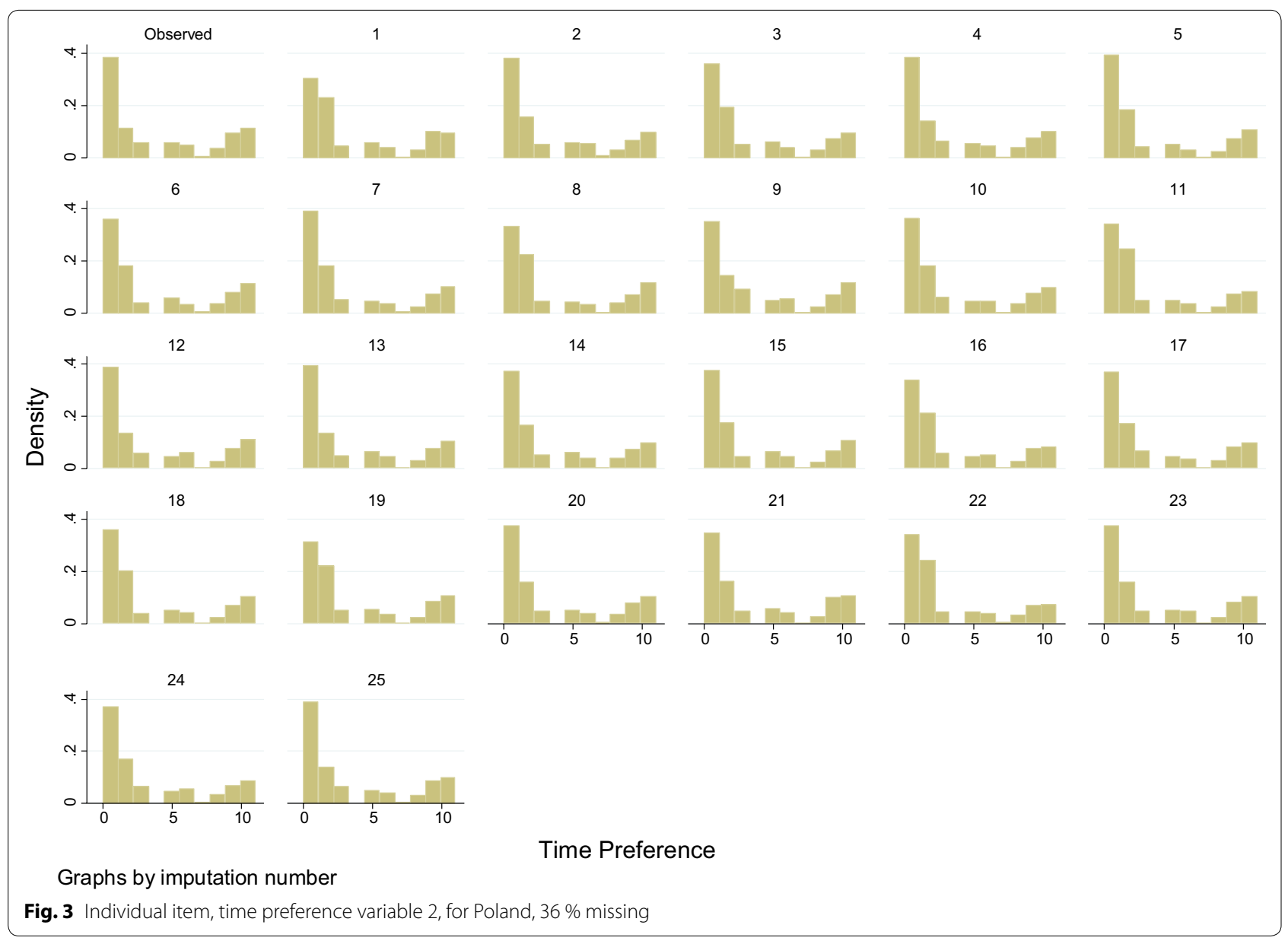

satisfaction, for methods 1,2 and 5 , with methods 5 and 6 showing significant bias on the slope. In terms of coverage, there are no significant differences between methods. Empirical standard error also shows little variability between methods, except that it is lower for method 5 on slope for practitioner satisfaction. This reflects the downward bias.

Increasing the number of incomplete observations with partial data, as in scenario 2 (Fig. 6) or increasing the number of incomplete observations (Fig. 7) indicate a similar story. Methods 1 and 2 show an increase in bias compared to the base case, with method 4 showing significant downward bias and reduced coverage for TPB slope in both scenarios. In both scenarios the empirical standard error appears lower than for the base case, reflecting downward bias.

Overall, method 6 is seen to be the best, broadly exhibiting the least bias and the most efficiency, and we regard it as a benchmark. This method is not always feasible however, for example in the case study described above. Method 1 often displays a large amount of bias, and like method 3, is inefficient and wasteful of observed data.
Method 2 indicates bias in all bar the base case, and may artificially reduce variability due to being effectively single imputation.

It appears therefore from the simulations and assumptions that in terms of bias, coverage and empirical standard error that method 4 or 5 would be best in cases where method 6 is not feasible. At this point it is unclear which of the two methods is most appropriate, method 4, similar to method 2 is akin to a single imputation, and for method 5 whilst the assumptions seem more appropriate, the simulation evidence suggests it can introduce bias when these are violated.

\section{Discussion}

Our proposed method for handling multiple multi-item scales allows imputation of individual items by using scale totals within the imputation models, such that given primary outcome $p$, scale $T$ and demographics $d_{1}$ $\ldots d_{\mathrm{n}}$, item $s_{1}$ from scale $S$, is imputed using $p, d_{1} \ldots d_{\mathrm{n}}$, $s_{2} \ldots s_{\mathrm{n}}$ and summary score $t$. The use of summed scale scores within the predictor equations reduces both the number of predictors in each equation and the sparsity 

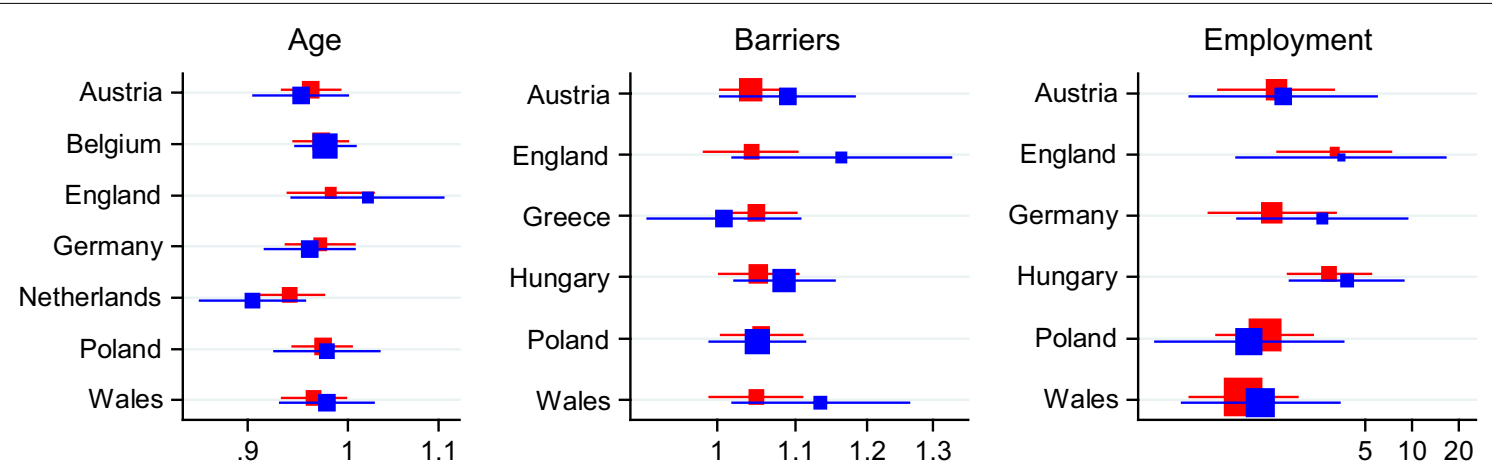

Personal Control

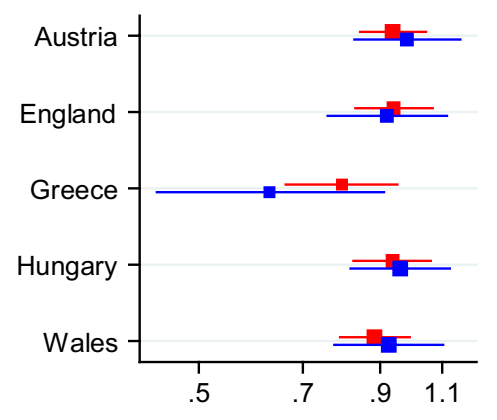

Self Efficacy

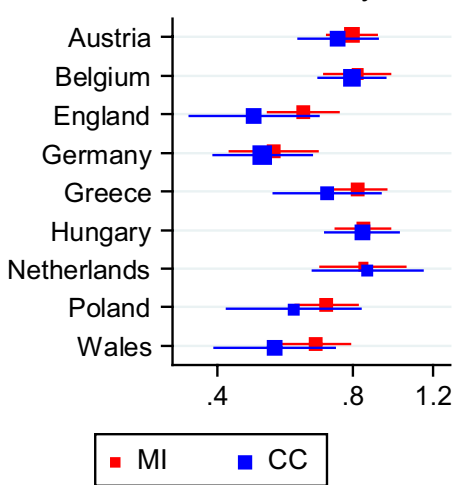

Fig. 4 Forest plots illustrating odds ratios from the logistic regression

Table 3 Summary of proportional decrease in standard error, between complete case and multiple imputation analyses

\begin{tabular}{lccccc}
\hline & Mean (\%) & Min (\%) & Max (\%) & $\begin{array}{l}\text { Median } \\
\text { (\%) }\end{array}$ & $\begin{array}{l}\text { Standard } \\
\text { deviation } \\
\text { (\%) }\end{array}$ \\
\hline Overall & 39 & 0 & 100 & 38 & 19 \\
Austria & 38 & 22 & 55 & 38 & 8 \\
Belgium & 5 & 0 & 13 & 4 & 5 \\
England & 58 & 33 & 100 & 45 & 27 \\
Germany & 21 & 12 & 26 & 22 & 5 \\
Greece & 50 & 43 & 58 & 50 & 4 \\
Hungary & 23 & 14 & 27 & 23 & 4 \\
Nether- & 29 & 24 & 36 & 29 & 4 \\
$\quad$ lands & & & & & \\
Poland & 41 & 14 & 59 & 42 & 12 \\
Wales & 36 & 28 & 47 & 37 & 6 \\
\hline
\end{tabular}

in the data set. This approach facilitates the efficient use of MI in large survey data sets with multiple multi-item scales. Using MICE allows preservation of the structure of the data, in terms of point estimates and variance or variables, and covariance. Should the approach presented here still result in overly complex prediction equations, a further simplification would be to replace $s_{2} \ldots s_{\mathrm{n}}$ in the prediction equation by their sum or average.

For subscales of the health psychology measures, rather than to impute every individual item, one simplification of our method would be to impute only the totals of the subscales. For our data this would reduce the size of the model from 134 to 56 predictors per variable. A disadvantage of this approach, however, is that it would restrict analysis to summed scales, leaving no scope for exploring individual items.

Forming scale totals prior to imputation, and then imputing missing totals is a further simplification, but comes with an additional disadvantage: for those respondents who have completed some, but not all, of the items in a subscale, those responses are discarded, or imputed by an ad-hoc method such as using the mean of observed items. Taking as an example the 17-item practitioner satisfaction scale in the Austrian data set, 262 respondents (from 323) completed all items. The response rate to individual items ranged from 278 to 292 responses, and the above approach would discard a total of 437 responses, collected from 31 respondents, from this scale.

Our simulation study compares these alternatives with a benchmark 'complete' MI analysis, and complete case 
Table 4 Disparity in variable selection between CC and MI, over 42 variables in 9 countries

\begin{tabular}{llllr}
\hline & & \multicolumn{2}{l}{ Complete case method } & Excluded n (\%) \\
\cline { 3 - 4 } & & Included $\mathbf{n}(\%)$ & $3(1)$ & Total \\
\hline Multiple imputation & Included $\mathrm{n}(\%)$ & $86(23)$ & $259(69)$ & 284 \\
& Excluded $\mathrm{n}(\%)$ & $25(7)$ & 262 & 373 \\
\hline
\end{tabular}

$x^{2}=250, p<0.001$
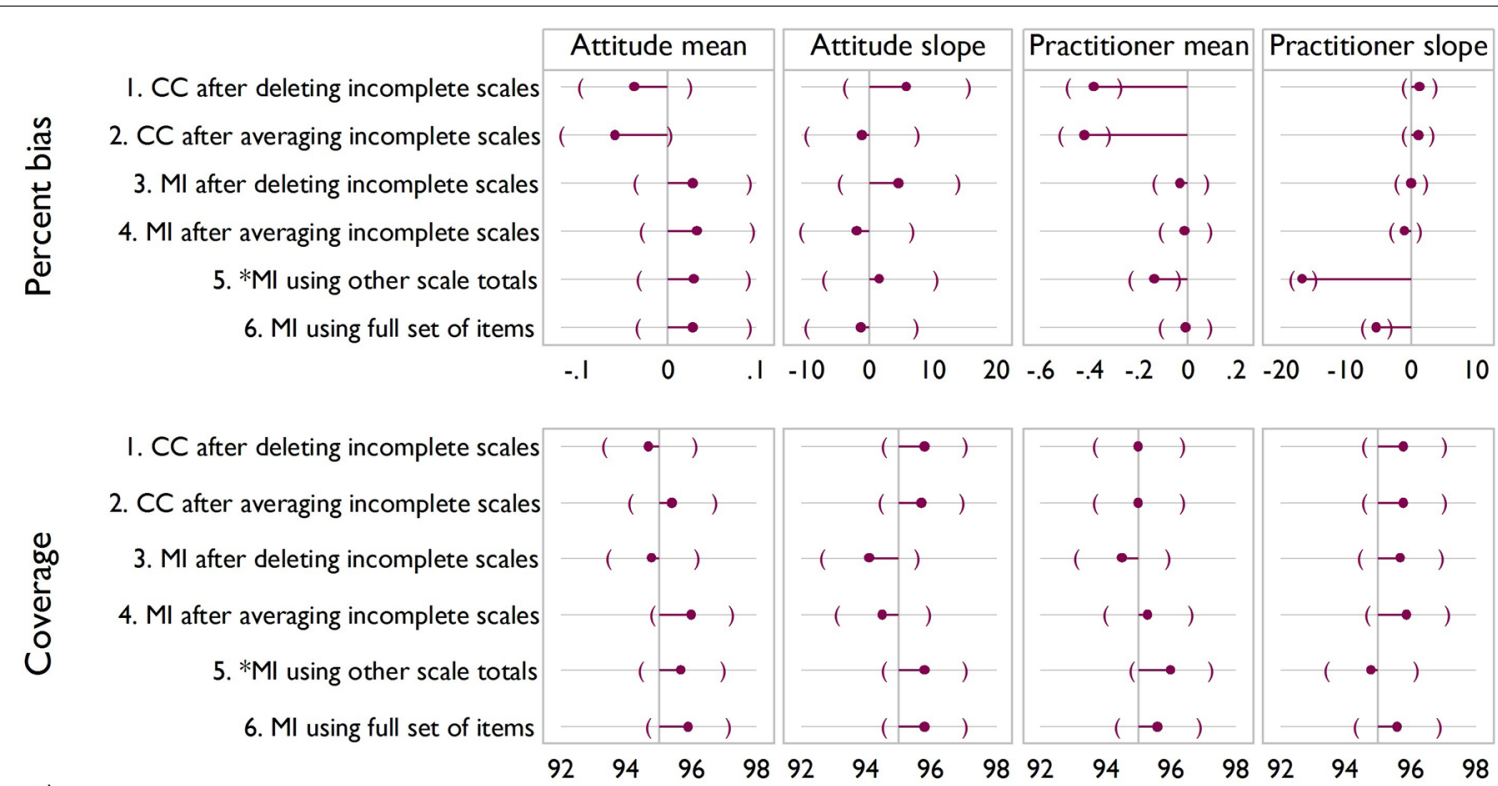

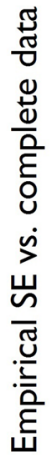

I. CC after deleting incomplete scales

2. CC after averaging incomplete scales

3. $\mathrm{Ml}$ after deleting incomplete scales

4. Ml after averaging incomplete scales

5. *Ml using other scale totals

6. Ml using full set of items
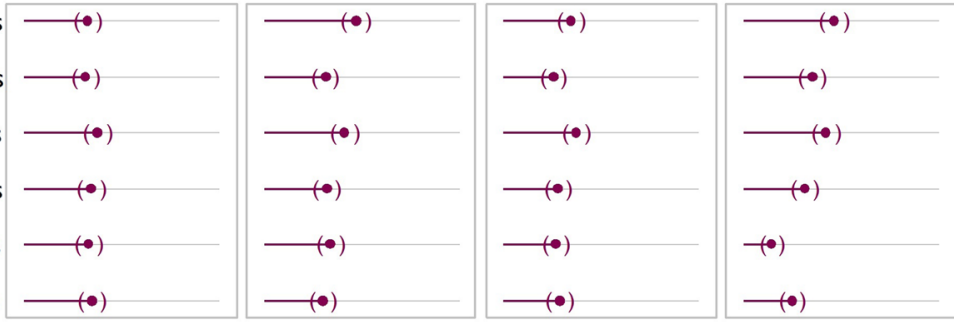

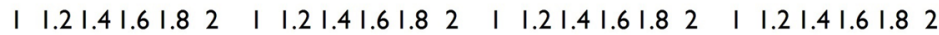

Fig. 5 Simulation results for the three scenarios. Brackets indicate confidence intervals. Base case: $35 \%$ had all items missing for a scale; $8 \%$ had one or two items missing

alternatives. Results from the simulation indicate that simplification by averaging incomplete scales and our proposed method perform comparably, with the simplification reducing model complexity, compromised with a slight loss of efficiency. Complete case methods were seen to perform poorly, with an increase in bias, particularly when the amount of missingness was increased. This result is consistent with a previous study on multiitem imputation, where mean imputation and single imputation were shown to have larger bias and worse coverage than item level multiple imputation, and complete case analysis was shown to overestimate standard error and reduce power [16].

Certain limitations are acknowledged. Typically, analysis with MI relies on an assumption of data being MAR. This assumption cannot be proven, but for large wellconducted surveys, the assumption of MAR is often considered a reasonable starting point for statistical analysis. 

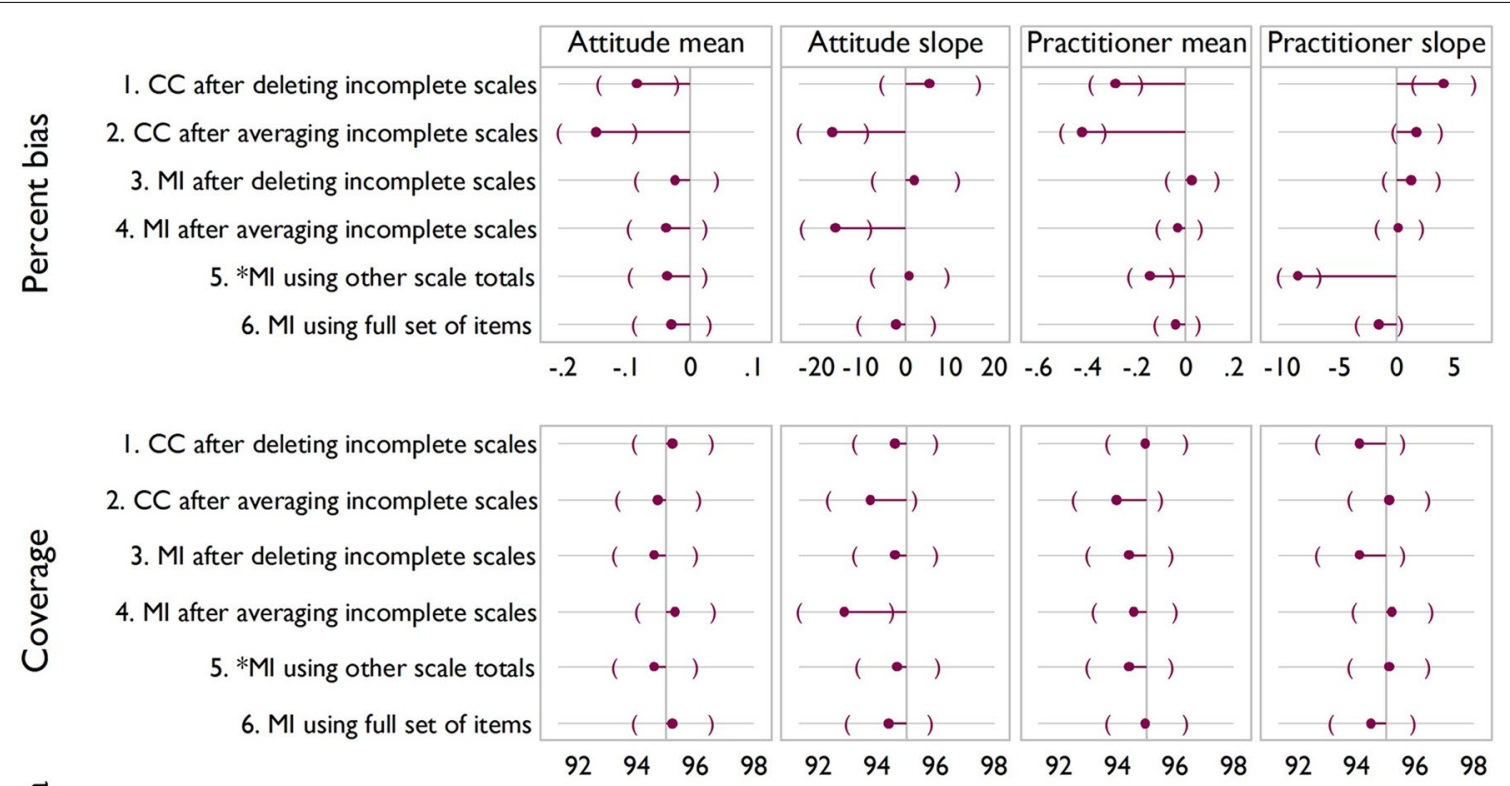

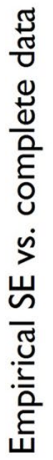

I. CC after deleting incomplete scales (०)

2. CC after averaging incomplete scales

3. $\mathrm{Ml}$ after deleting incomplete scales

4. Ml after averaging incomplete scales

5. *Ml using other scale totals

6. MI using full set of items
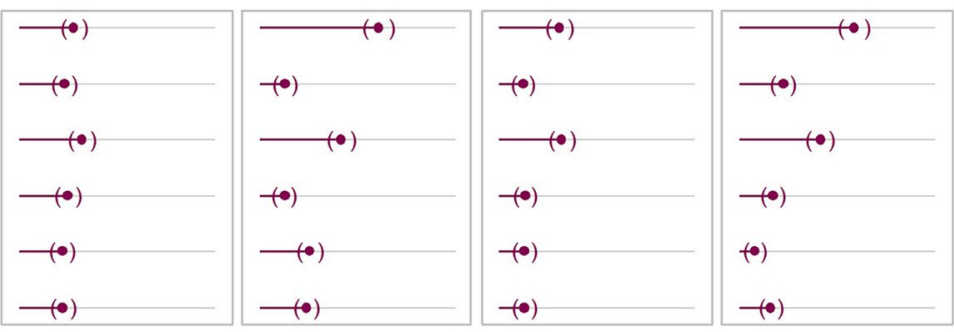

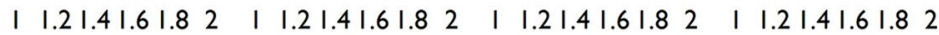

Fig. 6 Simulation results for the three scenarios. Brackets indicate confidence intervals. More incomplete observations with partial data: $18 \%$ had all items missing for a scale; $25 \%$ had one or two items missing

Rubin et al. conclude that whilst assuming MAR may be inadequate if there are high levels of missingness or insufficient relevant covariates, "the evidence seems to be that at least in some carefully designed survey contexts, modelling observed data under MAR can provide acceptable answers" [27].

A further limitation of the proposed method for handling multiple multi-item scales is the assumption that items from different scales are only correlated via the scale totals. The majority of scales and measures used in this survey are validated, and were tested during analysis for internal consistency. Additionally, as the survey was structured such that each measure was presented in its entirety, independently, this a plausible assumption for our data.

During data analysis, significant univariate differences were observed between $\mathrm{CC}$ and MI which indicate that performing variable selection on different data sets would result in the entry of different variables into the regression model. In comparison with $\mathrm{CC}$, using the same variables for both approaches, regression results from MI show standard error to be reduced by an average of $39 \%$, with no cases where standard error increases, thus resulting in more precise conclusions.

Data collection will almost always result in missing data. It is the duty of researchers and analysts to firstly minimise the extent of missing data by ensuring appropriate methods for enhancing data capture are implemented, but also to handle missingness in a way best suited to the data and research question. Poor handling and reporting of missing data may result in misleading conclusions and are one of the main reasons for publication rejections $[28,29]$. With the advent of MI routines in SPSS, R, SAS and Stata, MI is now readily accessible to analysts as a robust method for handling missing data, which can be applied in a number of contexts [4].

Alongside our proposed adaptation for imputing multiple multi-item scales, ordinal regression and conditional 

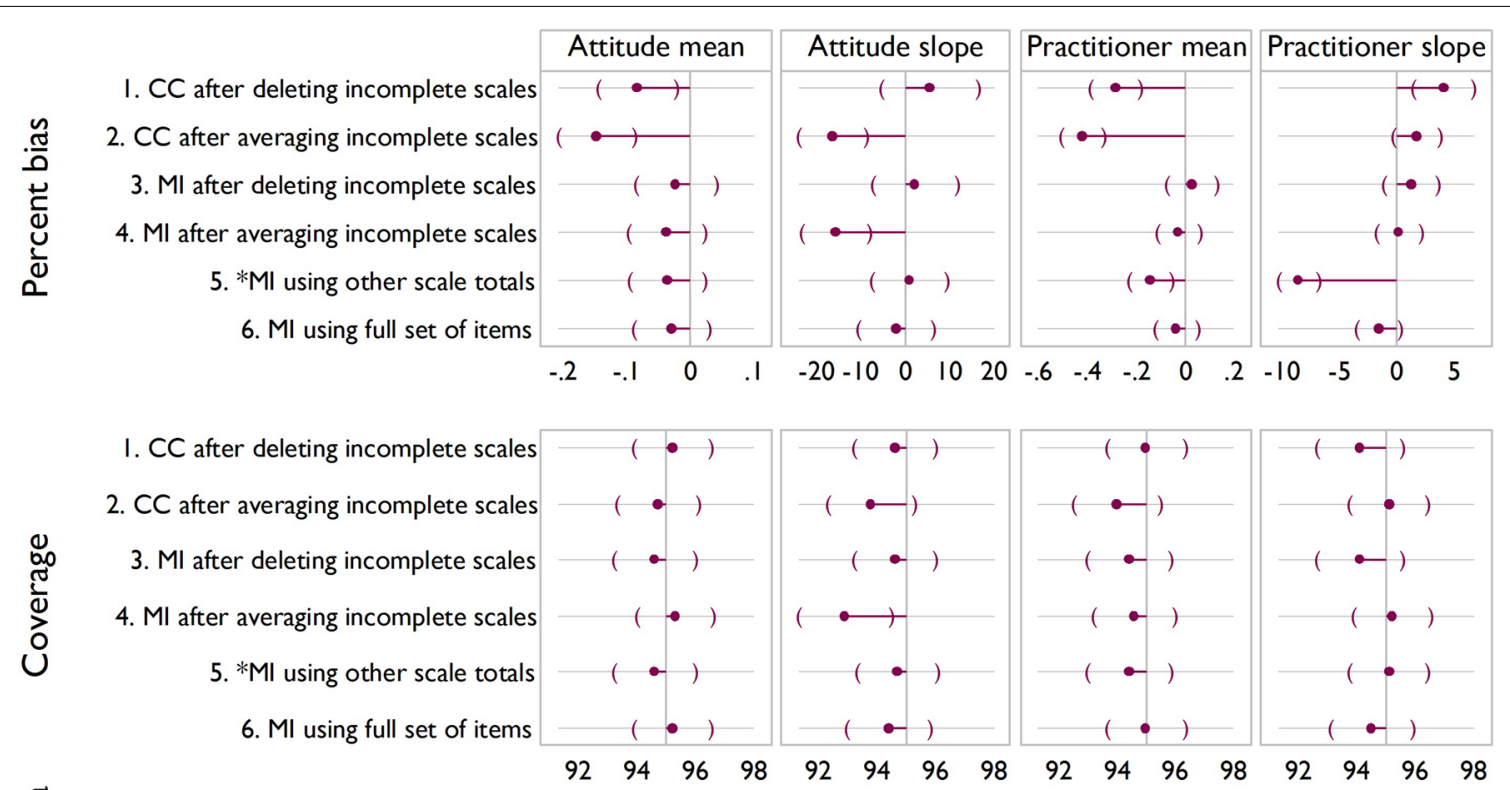

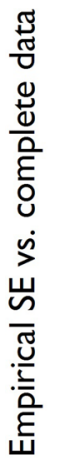

I. CC after deleting incomplete scales $\longrightarrow(\bullet)$

2. CC after averaging incomplete scales

3. MI after deleting incomplete scales

4. Ml after averaging incomplete scales
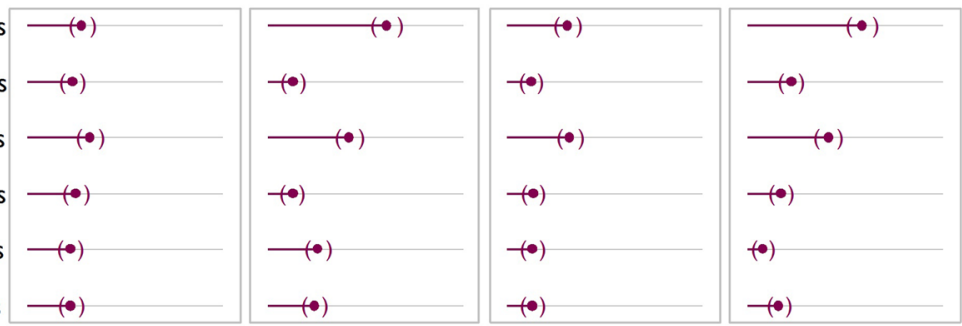

I I.2I.4I.6I.8 2

1.21 .41 .61 .82

1.21 .41 .61 .82

| 1.21 .41 .61 .82

Fig. 7 Simulation results for the three scenarios. Brackets indicate confidence intervals. Fewer observations with complete data: $55 \%$ had all items missing for a scale; $15 \%$ had just one or two missing

imputation are also powerful tools which, in this study, have allowed us to address the challenges presented by large scale survey data. Our proposed adaptation makes MI practical for large scale survey data, where a full prediction model may be infeasible, and we have shown that the use of MI in this way makes better use of available data and can yield substantively different results from simpler techniques.

\section{Additional files}

Additional file1. Stata code.

Additional file 2. Responses to income questions.

\section{Authors' contributions}

$\mathrm{DH}$ conceived the research. CP, IW contributed to the design, analysis and interpretation of data. TM designed and implemented the simulation study, CP drafted the article and all authors revised it critically for important intellectual content, and gave their final approval of the version to be published. All authors read and approved the final manuscript.

\section{Author details}

${ }^{1}$ Centre for Health Economics and Medicines Evaluation, Bangor University, Ardudwy, Normal Site, Holyhead Road, Bangor, Gwynedd LL57 2PZ, UK. ${ }^{2}$ MRC Clinical Trials Unit at UCL, Institute of Clinical Trials and Methodology, Aviation House, 125 Kingsway, London WC2B 6NH, UK. ${ }^{3}$ London School of Hygiene and Tropical Medicine, Keppel Street, London WC1E 7HT, UK. ${ }^{4}$ MRC Biostatistics Unit, Cambridge Institute of Public Health, Robinson Way, Cambridge CB2 OSR, UK.

\section{Acknowledgements}

We are grateful to Emily Fargher, Dr Valerie Morrison, Dr Sahdia Parveen and other members of the ABC Project Team for their support on this project.

\section{Competing interests}

The authors declare that they have no competing interests.

\section{Funding}

This work was supported by: the European Union's Seventh Framework Programme FP7/2007-2013 "Ascertain Barriers for Compliance (ABC) project" under grant agreement number 223477; DH is supported by the Medical Research Council North-West Hub in Trials Methodology Research, reference 
number G0800792. IW was supported by the Medical Research Council [Unit Programme number U105260558].

\section{Role of the funder}

The funder had no role in the study design; in the collection, analysis, and interpretation of data; in the writing of the report; or in the decision to submit the paper for publication.

Received: 17 December 2015 Accepted: 12 January 2016

Published online: 26 January 2016

\section{References}

1. White IR, Royston P, Wood AM. Multiple imputation using chained equations: issues and guidance for practice. Stat Med. 2011;30(4):377-99.

2. Wayman JC. Multiple imputation for missing data: What is it and how can I use it? In Proceedings of the Annual meeting of the American Educational Research Association Chicago, II. 2003.

3. Baraldi AN, Enders CK. An introduction to modern missing data analyses. J Sch Psychol. 2010;48:5-37.

4. Roth PL. Missing Data: a conceptual review for applied psychologists. Personnel Psychology. 1994;41(3):537-60.

5. Wood A, White IR, Thompson SG. Are missing outcome data adequately handled? a review of published randomized controlled trials in major medical journals. Clin Trials. 2004;1:368-76.

6. Peugh $J$, Enders CK. Missing data in educational research: a review of reporting practices and suggestions for improvement. Rev Educ Res. 2004;74(4):525-56.

7. Little RJA. Missing-data adjustments in large surveys. J Bus Econ Stat. 1988;6(3):287-96.

8. Sinharay S, Stern HS, Russell D. The use of multiple imputation for the analysis of missing data. Psychol Methods. 2001;6(4):317-29.

9. Sterne JAC, White IR, Carlin JB, et al. Multiple imputation for missing data in epidemiological and clinical research: potential and pitfalls. BMJ. 2009:338:b2393

10. Noble SM, Hollingworth W, Tilling K. Missing data in trial-based cost-effectiveness. analysis: the current state of play. Health Econ. 2012;21(2):187-200.

11. Rousseau M, Simon M, Bertrand R, et al. Reporting missing data: a study of selected articles published from 2003-2007. Qual Quant. 2012;46(5):1393-406.

12. Karahalios A, Baglietto L, Carlin JB, et al. A review of the reporting and handling of missing data in cohort studies with repeated assessment of exposure measures. BMC Med Res Methodol. 2012;12:96.
13. Eekhout I, de Boer MR, Twisk JWR, et al. Missing Data: a systematic review of how they are reported and handled. Epidemiology. 2012;23(5):729-32.

14. Bell ML, Fiero M, Horton NJ, et al. Handling missing data in RCTs; a review of the top medical journals. BMC Med Res Methodol. 2014;14:118.

15. Simons $C L$, Rivero-Arias $O$, Yu LM, et al. Multiple imputation to deal with missing EQ-5D-3L data: should we impute individual domains or the actual index? Qual Life Res. 2015;24:805-15.

16. Eekhout I, de Vet HCW, Twisk JWR, et al. Missing data in a multi-item instrument were best handled by multiple imputation at the item score level. J Clin Epidemiol. 2014;67:335-42.

17. van Buuren $\mathrm{S}$, Oudshoorn CGM. Multiple imputation by chained equations: MICE V1.0 user's manual. TNO Report PG/NGZ/00.038. TNO Preventie enGezondheid: Leiden (2000). http://www.multiple-imputation.com/ Accessed 26 Nov 2012.

18. White IR, Daniel R, Royston P. Avoiding bias due to perfect prediction in multiple imputation of incomplete categorical variables. Comput Stat Data Anal. 2010;54:2267-75.

19. Royston P. Multiple imputation of missing values: update. Stata J. 2005;5(2):188-201.

20. Rubin DB. Multiple imputation for nonresponse in surveys. New York: Wiley; 1987.

21. Morrison VL, Holmes EAF, Parveen S, et al. Predictors of self-reported adherence to antihypertensive medicines: a multi-national, cross-sectional survey. Value Health. 2015. doi:10.1016/j.val.2014.12.013.

22. Morisky DE, Ang A, Krousel-Wood M, et al. Predictive validity of a medication adherence measure for hypertension control. J Clin Hypertens. 2008;10(5):348-54.

23. van der Pola M, Cairns J. Comparison of two methods of eliciting time preference for future health states. Soc Sci Med. 2008;67(5):883-9.

24. Royston P. Multiple imputation of missing values: update of ice. Stata J. 2005;5(4):527-36

25. Royston P. Multiple imputation of missing values: further update of ice, with an emphasis on categorical variables. Stata J. 2009:9(3):466-77.

26. Wood AM, White IR, Royston P. How should variable selection be performed with multiply imputed data? Stat Med. 2008;27:3227-46.

27. Rubin DB, Stern HS, Vehovar V. Handling, "don't know" survey responses: the case of the slovenian plebiscite. J Am Stat Assoc. 1995;90:822-8.

28. Fernandes-Taylor S, Hyun JK, Reeder RN, et al. Common statistical and research design problems in manuscripts submitted to high-impact medical journals. BMC Res Notes. 2011:4:304.

29. Harris A, Reeder R, Hyun J. Survey of editors and reviewers of high-impact psychology journals: statistical and research design problems in submitted manuscripts. J Psychol. 2011;145(3):195-209.

\section{Submit your next manuscript to BioMed Central and we will help you at every step:}

- We accept pre-submission inquiries

- Our selector tool helps you to find the most relevant journal

- We provide round the clock customer support

- Convenient online submission

- Thorough peer review

- Inclusion in PubMed and all major indexing services

- Maximum visibility for your research

Submit your manuscript at www.biomedcentral.com/submit
( Biomed Central 